\title{
SOME PROPERTIES ON ISOLOGISM OF GROUPS
}

\author{
MOHAMMAD REZA R. MOGHADDAM and ALI REZA SALEMKAR
}

(Received 4 September 1997; revised 16 February 1999)

\author{
Communicated by R. B. Howlett
}

\begin{abstract}
In this paper a necessary and sufficient condition will be given for groups to be $\mathcal{V}$-isologic, with respect to a given variety of groups $\mathcal{V}$. It is also shown that every $\mathcal{V}$-isologism family of a group contains a $\mathcal{V}$-Hopfian group. Finally we show that if $G$ is in the variety $\mathcal{V}$, then every $\mathcal{V}$-covering group of $G$ is a Hopfian group.
\end{abstract}

1991 Mathematics subject classification (Amer. Math. Soc.): primary 20E10, 20E36; secondary $20 \mathrm{~F} 28$. Keywords and phrases: covering group, isologism, Hopfian group.

\section{Introduction and preliminary results}

Let $F_{\infty}$ be the free group freely generated by a countable set $\left\{x_{1}, x_{2}, \ldots\right\}$ and let $V$ be a subset of $F_{\infty}$. Let the variety of groups $\mathcal{V}$ be defined by the set of laws $V$. It is assumed that the reader is familiar with the notion of the verbal subgroup, $V(G)$, and the marginal subgroup, $V^{*}(G)$, associated with the variety $\mathcal{V}$ and a given group $G$. See Neumann [8] for more information on varieties of groups.

In 1940, Hall [1] introduced the notion of isoclinism and then he extended it to the notion of $\mathcal{V}$-isologism, with respect to a given variety of groups $\mathcal{V}$. If $\mathcal{V}$ is the variety of Abelian or nilpotent groups of class at most $n$, then $\mathcal{V}$-isologism coincides with isoclinism and $n$-isoclinism properties, respectively (see $[1,2])$.

In the next section we define some closure operation with respect to a variety of groups $\mathcal{V}$, and show that a group $G$ is $\mathcal{V}$-isologic to a group $H$ (written by $G \widetilde{\mathcal{V}} H$ ) if and only if $G$ and $H$ have the same $\mathcal{V}$-closure (see Theorem 2.5).

Finally, if $H_{1}$ and $H_{2}$ are two $\mathcal{V}$-covering groups of a given group $G$ and $f$ is an epimorphism of $H_{1}$ onto $H_{2}$ with some other condition, then $f$ is an isomorphism.

(C) 2000 Australian Mathematical Society $0263-6115 / 2000 \$ A 2.00+0.00$ 
From this result we conclude that all $\mathcal{V}$-covering groups of an arbitrary group in the variety $\mathcal{V}$ are Hopfian.

In the following we recall the definitions of isologism and the Hopf property of groups.

DEFINITION 1.1. Let $\mathcal{V}$ be a variety of groups defined by the set of laws $V$, and let $G$ and $H$ be two groups. Then the pair $(\alpha, \beta)$ is said to be a $\mathcal{V}$-isologism between the groups $G$ and $H$, if the maps

$$
\begin{aligned}
\alpha: G / V^{*}(G) & \longrightarrow H / V^{*}(H), \\
\beta: V(G) & \longrightarrow V(H)
\end{aligned}
$$

are isomorphisms such that for all words $v\left(x_{1}, \ldots, x_{r}\right)$ in $V$ and all the elements $g_{1}, \ldots, g_{r}$ in $G$, we have

$$
\beta\left(v\left(g_{1}, \ldots, g_{r}\right)\right)=v\left(h_{1}, \ldots, h_{r}\right),
$$

whenever $h_{i} \in \alpha\left(g_{i} V^{*}(G)\right)$, for $i=1,2, \ldots, r$. In this case we write $G \widetilde{\mathcal{V}} H$ and say that the group $G$ is $\mathcal{V}$-isologic to $H$.

A group $G$ is said to be a Hopfian group, if every epimorphism $G \rightarrow G$ is an isomorphism, otherwise $G$ is non-Hopfian.

Clearly isologism is an equivalence relation, and hence gives rise to a partition on the class of all groups into equivalence classes, the so called isologism families.

One notes that if $A$ is any group belonging to the variety $\mathcal{V}$, then $G \times A \widetilde{\mathcal{V}} G$, for all groups $G$.

The proof of the following lemma is straightforward (see also Hekster [3]).

LEMMA 1.2. Let $\mathcal{V}$ be a variety of groups and $H$ be a subgroup and $N$ be a normal subgroup of a group $G$. Then the following statements hold:

(i) $H \widetilde{\mathcal{V}} H V^{*}(G)$. In particular, if $G=H V^{*}(G)$ then $G \widetilde{\mathcal{V}} H$. Conversely, if the marginal factor group $G / V^{*}(G)$ satisfies the descending chain condition on subgroups and $G \tilde{\mathcal{V}} H$, then $G=H V^{*}(G)$.

(ii) $G / N \widetilde{v} G / N \cap V(G)$. In particular, if $N \cap V(G)=\langle 1\rangle$, then $G \widetilde{v} G / N$. Conversely, if $V(G)$ satisfies the ascending chain condition on normal subgroups and $G \tilde{\mathcal{V}} G / N$, Then $N \cap V(G)$ is trivial.

Now, in the spirit of the above Lemma 1.2 (ii), we introduce the following

DEFINITION 1.3. Let $\mathcal{V}$ be a variety of groups defined by the set of laws $V$. A group $G$ is said to be $\mathcal{V}$-Hopfian, with respect to $\mathcal{V}$-isologism, if $G$ contains no non-trivial normal subgroup $N$ satisfying $N \cap V(G)=\langle 1\rangle$. 


\section{2. $\mathcal{V}$-isologism of groups}

Let $\mathcal{V}$ be a variety of groups defined by the set of laws $V$. A group $G$ is called $\mathcal{V}$-marginal group, if $G=V^{*}(G)$.

Now, in the following we define a $\mathcal{V}$-closure operation similar to [9], which is done for the variety of Abelian groups.

DEFINITION 2.1. Let $G$ be a group. Then $\{G\}_{\mathcal{V}}$ denotes the smallest class of groups containing $G$, closed under the operation of forming direct products with $\mathcal{V}$-marginal groups, and satisfying the following property: if $H \in\{G\}_{\mathcal{V}}$ then every subgroup $K$ of $H$ which satisfies $H=K V^{*}(H)$ is also in $\{G\}_{\mathcal{V}}$, and for every normal subgroup $N$ of $H$ which satisfies $N \cap V(H)=\langle 1\rangle$ the quotient group $H / N$ is also in $\{G\}_{\mathcal{V}}$. We call the set $\{G\}_{\mathcal{V}}$ the $\mathcal{V}$-closure of $G$.

One should note that we may replace the group $G$ by a set of groups $\left\{G_{i}\right\}$, thus obtaining a $\mathcal{V}$-closure operator for sets of groups.

The following proposition can be proved easily.

PROPOSITION 2.2. Let $\left\{G_{i}\right\}$ and $\left\{H_{j}\right\}$ be two sets of groups. Then

(a) $\left\{G_{i}\right\} \subseteq\left\{G_{i}\right\}_{\mathcal{V}}$.

(b) $\left\{\left\{G_{i}\right\}_{\mathcal{V}}\right\}_{\mathcal{V}}=\left\{G_{i}\right\}_{\mathcal{V}}$.

(c) if $\left\{G_{i}\right\} \subseteq\left\{H_{j}\right\}$, then $\left\{G_{i}\right\}_{\mathcal{V}} \subseteq\left\{H_{j}\right\}_{\mathcal{V}}$.

The following result yields the necessary tools for our main result (Theorem 2.6).

THEOREM 2.3. Let $G$ and $H$ be two groups. Then $G$ and $H$ are $\mathcal{V}$-isologic if and only if a group $C$ and subgroups $V_{G}^{*}, V_{H}^{*}$ of $C$ exist such that $G \cong C / V_{H}^{*}, H \cong C / V_{G}^{*}$ and the following equivalent statements hold:

(a) $G \cong C / V_{H}^{*} \widetilde{\mathcal{v}} C \widetilde{\mathcal{v}} C / V_{G}^{*} \cong H$;

(b) $C / V_{H}^{*} \times C / V(C) \widetilde{\mathcal{v}} C_{H} \cong C \cong C_{G} \widetilde{\mathcal{v}} C / V_{G}^{*} \times C / V(C)$,

for some subgroup $C_{H}$ of $C / V_{H}^{*} \times C / V(C)$ and some subgroup $C_{G}$ of $C / V_{G}^{*} \times C / V(C)$.

Proof. It is clear that if such groups $C, V_{G}^{*}$ and $V_{H}^{*}$ exist then $G \widetilde{\mathcal{V}} H$.

Conversely, let $G \tilde{\mathcal{V}} H$, and $(\alpha, \beta)$ be a $\mathcal{V}$-isologism between the groups $G$ and $H$. Assume

$$
\begin{aligned}
C & =\left\{(g, h) \in G \times H \mid \alpha\left(g V^{*}(G)\right)=h V^{*}(H)\right\}, \\
V_{G}^{*} & =\left\{(x, 1) \in G \times H \mid x \in V^{*}(G)\right\}, \\
V_{H}^{*} & =\left\{(1, y) \in G \times H \mid y \in V^{*}(H)\right\} .
\end{aligned}
$$


Clearly, $V_{G}^{*} \cong V^{*}(G)$ and $V_{H}^{*} \cong V^{*}(H)$. Define the map $\varphi$ from $C$ into $G$ by $\varphi(g, h)=g$. It is easy to see that $\varphi$ is an epimorphism with $\operatorname{ker} \varphi=V_{H}^{*}$. Hence $C / V_{H}^{*} \cong G$. Similarly $C / V_{G}^{*} \cong H$.

(a) The verbal subgroup $V(C)$ is generated by

$$
\left\{\left(\nu\left(g_{1}, \ldots, g_{r}\right), \beta\left(\nu\left(g_{1}, \ldots, g_{r}\right)\right)\right) \mid g_{1}, \ldots, g_{r} \in G, v \in V\right\} .
$$

Clearly, $V(C) \cap V_{H}^{*}=\langle 1\rangle$, for if $(g, h) \in V(C) \cap V_{H}^{*}$ then $g=1$ and hence $h=\beta(1)=1$. Similarly $V(C) \cap V_{G}^{*}$ is also trivial. Thus by Lemma 1.2 (ii),

$$
C / V_{G}^{*} \widetilde{v} C \widetilde{v} C / V_{H}^{*},
$$

which proves part (a).

(b) We define the subgroup $C_{G}$ of $C / V_{G}^{*} \times C / V(C)$ to be

$$
C_{G}=\left\{\left(x V_{G}^{*}, x V(C)\right) \mid x \in C\right\} .
$$

It is clear that the map $\psi: C \rightarrow C_{G}$, given by $\psi(x)=\left(x V_{G}^{*}, x V(C)\right)$, defines an isomorphism and hence $C \cong C_{G}$. Now, in view of Lemma 1.2 (i), to show

$$
C / V_{G}^{*} \times C / V(C) \widetilde{\mathcal{V}} C_{G}
$$

it is enough to prove that $C / V_{G}^{*} \times C / V(C)=C_{G} V^{*}\left(C / V_{G}^{*} \times C / V(C)\right)$. Let $a=$ $\left(x V_{G}^{*}, y V(C)\right)$ be an arbitrary element of $C / V_{G}^{*} \times C / V(C)$. Clearly $a=b c$, where $b=\left(x V_{G}^{*}, x V(C)\right) \in C_{G}$ and $c=\left(V_{G}^{*}, x^{-1} y V(C)\right)$. It is easily seen that

$$
c \in V^{*}\left(C / V_{G}^{*} \times C / V(C)\right) .
$$

This implies that

$$
C / V_{G}^{*} \times C / V(C) \subseteq C_{G} V^{*}\left(C / V_{G}^{*} \times C / V(C)\right) .
$$

The reverse containment follows immediately. Hence

$$
C \cong C_{G} \widetilde{v} C / V_{G}^{*} \times C / V(C) .
$$

By a similar argument it follows that

$$
C \cong C_{H} \widetilde{v} C / V_{H}^{*} \times C / V(C),
$$

in which $C_{H}=\left\{\left(y V_{H}^{*}, y V(C) \mid y \in C\right\}\right.$.

The following corollary generalizes a result of Weichsel [9] to an arbitrary variety of groups. 
COROLLARY 2.4. Let $G$ and $H$ be two groups and $\mathcal{V}$ be a variety of groups. Then $G \widetilde{\mathcal{V}} H$ if and only if there exists a $\mathcal{V}$-marginal group $K$, a subgroup $L$ of $G \times K$ with $L V^{*}(G \times K)=G \times K$ and a normal subgroup $N$ of $L$ such that $N \cap V(L)=1$ and $H \cong L / N$.

Proof. Assume that $G \widetilde{\mathcal{V}} H$, then the result follows from the above theorem by taking $K=C / V(C), L=C_{H}$, and $N=V_{G}^{*}$.

Conversely, suppose the required groups exist, then it follows immediately that $H \widetilde{\mathcal{V}} L \widetilde{\mathcal{v}} G \times K \widetilde{\mathcal{v}} G$

Using the notation as in Definition 2.1 we obtain the following.

THEOREM 2.5. $\{G\}_{\mathcal{V}}$ is the $\mathcal{V}$-isologism family of the group $G$, and hence $G \widetilde{\mathcal{V}} H$ if and only if $\{G\}_{\mathcal{V}}=\{H\}_{\mathcal{V}}$.

Proof. Clearly the $\mathcal{V}$-isologism family of the group $G$ contains $G$ and it is closed under the operations given in Definition 2.1, and hence it contains $\{G\}_{\mathcal{V}}$. But by Corollary 2.4 , any group isologic to $G$ can be constructed from $G$ using the allowable operations of $\{G\}_{\mathcal{V}}$, and so is contained in $\{G\}_{\mathcal{V}}$.

Finally, in this section we show that for any group $G$, the set $\{G\}_{\mathcal{V}}$ contains a group, $H$ say, which is $\mathcal{V}$-Hopfian with respect to $\mathcal{V}$-isologism.

THEOREM 2.6. Let $G$ be a group. Then there exists a normal subgroup $N$ of $G$ such that $G \widetilde{\mathcal{V}} G / N$ and $G / N$ is $\mathcal{V}$-Hopfian.

ProOF. Let $\mathscr{N}=\{N \unlhd G \mid N \cap V(G)=\langle 1\rangle\}$. Clearly the set $\mathscr{N}$ is non-void, as it contains the trivial subgroup. We define a partial ordering on $\mathscr{N}$ by inclusion and clearly by Zorn's Lemma we can find a maximal normal subgroup $N$ in $\mathscr{N}$. Since $N \cap V(G)=\langle 1\rangle$, it follows, by Lemma 1.2, that $G \widetilde{\mathcal{V}} G / N$. Now, suppose there exists $M / N \unlhd G / N$ such that $M / N \cap V(G / N)=\langle 1\rangle$. By [3, Proposition 2.3] and Dedekind's modular law, we have $M \cap V(G) \subseteq N$. Since $N \cap V(G)=\langle 1\rangle$, it follows that $M \in \mathscr{N}$. On the other hand, we have $N \subseteq M$, so by the maximality of $N$, it follows that $M=N$. Therefore $M / N$ is trivial, and hence $G / N$ is $\mathcal{V}$-Hopfian with respect to $\mathcal{V}$-isologism.

\section{Hopfian property}

Let $H_{1}$ and $H_{2}$ be two $\mathcal{V}$-covering groups of a given group $G$. In this final section we give a sufficient condition for an epimorphism of $H_{1}$ onto $H_{2}$ to be an isomorphism. 
Then we conclude that every $\mathcal{V}$-covering group of a group in the variety $\mathcal{V}$ has the Hopf property.

Let $1 \rightarrow R \rightarrow F \stackrel{\pi}{\longrightarrow} G \rightarrow 1$ be a free presentation of a group $G$, where $F$ is a free group and $R=\operatorname{ker} \pi$. Then the Baer-invariant of $G$ with respect to the variety $\mathcal{V}$, denoted by $\mathcal{V} M(G)$, is defined to be $R \cap V(F) /\left[R V^{*} F\right]$, where $V(F)$ is the verbal subgroup of $F$ and $\left[R V^{*} F\right]$ is the least normal subgroup $T$ of $F$ contained in $R$ such that $R / T \subseteq V^{*}(F / T)$. One may check that the Baer-invariant of a group $G$ is always Abelian and independent of the choice of the free presentation of $G$. In particular, if $\mathcal{V}$ is the variety of Abelian or nilpotent groups of class at most $c(c \geq 1)$, then the Baerinvariant of the group $G$ will be $\left(R \cap F^{\prime}\right) /[R, F]$, which is the Schur-multiplicator of $G$, or $\left(R \cap \gamma_{c+1}(F)\right) /\left[R,{ }_{c} F\right]$ (where $F$ repeated $c$ times), respectively (see [4]).

We recall that an exact sequence $1 \rightarrow A \rightarrow G^{*} \rightarrow G \rightarrow 1$ is called a $\mathcal{V}$-stem extension with respect to the variety of groups $\mathcal{V}$, when $A \subseteq V\left(G^{*}\right) \cap V^{*}\left(G^{*}\right)$. If in addition $A \cong \mathcal{V} M(G)$, then the above extension is called a $\mathcal{V}$-stem cover. In this case $G^{*}$ is said to be a $\mathcal{V}$-covering group of $G$. It is of interest to know the class of groups that do not have $\mathcal{V}$-covering groups (see [7]). In [6] we have also shown that a given group $G$ has always a $\mathcal{V}$-covering group with respect to some specific variety $\mathcal{V}$. So whenever we talk about a $\mathcal{V}$-covering of a group, it is assumed that $\mathcal{V}$ is a suitable variety.

The following results of [5] are needed to prove the main result of this section.

THEOREM 3.1 (Moghaddam and Salemkar [5]). Let $\mathcal{V}$ be a variety of groups defined by the set of laws $V$, and let $1 \rightarrow R \rightarrow F \rightarrow G \rightarrow 1$ be a free presentation of a group $G$. Then

(i) If $S$ is a normal subgroup of $F$ such that

$$
\frac{R}{\left[R V^{*} F\right]}=\frac{R \cap V(F)}{\left[R V^{*} F\right]} \times \frac{S}{\left[R V^{*} F\right]},
$$

then $G^{*}=F / S$ is a $\mathcal{V}$-covering group of $G$.

(ii) Every $\mathcal{V}$-covering group of $G$ is a homomorphic image of $F /\left[R V^{*} F\right]$.

(iii) For any $\mathcal{V}$-covering group $G^{*}$ of $G$ with an exact sequence $1 \rightarrow A \rightarrow G^{*} \rightarrow$ $G \rightarrow 1$, such that $A \subseteq V^{*}\left(G^{*}\right) \cap V\left(G^{*}\right)$ and $A \cong \mathcal{V} M(G)$, then there exists a normal subgroup $S$ of $F$, as in (i), such that $F / S \cong G^{*}$ and $R / S \cong A$.

COROLLARY 3.2. With the above assumption, for any $\mathcal{V}$-covering group $G^{*}$ of a given group $G$, there exists an epimorphism $\bar{\psi}$ from $F /\left[R V^{*} F\right]$ onto $G^{*}$ such that

$$
\frac{R}{\left[R V^{*} F\right]}=\frac{R \cap V(F)}{\left[R V^{*} F\right]} \times \operatorname{ker} \bar{\psi}
$$

where the image under $\bar{\psi}$ of the first factor is equal to $A$. 
The following lemma is needed for the proof of Theorem 3.4 below, which is the main result of this section.

LEMMA 3.3. Let $G$ be a group, and

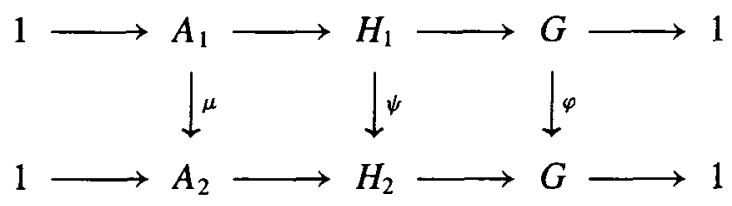

a commutative diagram of groups such that the first row is exact and the second one is a $\mathcal{V}$-stem extension of $G$. If the homomorphism $\varphi$ is onto, then so is $\psi$.

PROOF. It is easily shown that $H_{2}=(\operatorname{Im} \psi) A_{2}$. Hence by [3, Theorem 2.4],

$$
V\left(H_{2}\right)=V(\operatorname{Im} \psi)\left[A_{2} V^{*} H_{2}\right]
$$

But $A_{2} \subseteq V^{*}\left(H_{2}\right)$, by the assumption. Thus $V\left(H_{2}\right)=V(\operatorname{Im} \psi)$. We also have $A_{2} \subseteq V\left(H_{2}\right)$, which implies that $A_{2} \subseteq V(\operatorname{Im} \psi) \subseteq \operatorname{Im} \psi$, and hence $H_{2}=\operatorname{Im} \psi$.

THEOREM 3.4. Let $G$ be a group and let

$$
1 \longrightarrow A_{i} \longrightarrow H_{i} \longrightarrow G \longrightarrow 1, \quad i=1,2
$$

be two $\mathcal{V}$-stem covers of $G$ with respect to the variety $\mathcal{V}$. If $\psi: H_{1} \rightarrow H_{2}$ is an epimorphism such that $\psi\left(A_{1}\right)=A_{2}$, then $\psi$ is an isomorphism.

PROOF. Let $1 \rightarrow R \rightarrow F \rightarrow G \rightarrow 1$ be a free presentation of the group $G$. By Theorem 3.1 (iii), there exist normal subgroups $S_{i}$ of $F, i=1,2$, such that $H_{i} \cong F / S_{i}$ and $A_{i} \cong R / S_{i}$, and

$$
\frac{R}{\left[R V^{*} F\right]}=\frac{R \cap V(F)}{\left[R V^{*} F\right]} \times \frac{S_{i}}{\left[R V^{*} F\right]}
$$

So we may regard $\psi$ as an epimorphism from $F / S_{1}$ onto $F / S_{2}$ such that $\psi\left(R / S_{1}\right)=$ $R / S_{2}$. Therefore, by Corollary 3.2, there exists an epimorphism $\varphi: F /\left[R V^{*} F\right] \rightarrow$ $F / S_{2}$ such that $\operatorname{ker} \varphi=S_{2} /\left[R V^{*} F\right]$ and the following diagram is commutative

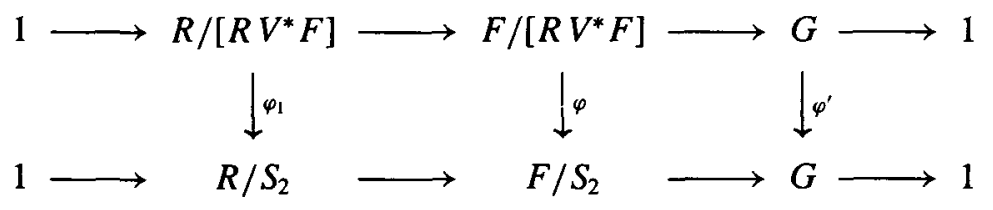


where $\varphi_{1}$ and $\varphi^{\prime}$ are the restriction and the induced homomorphisms of $\varphi$, respectively. One can easily check that $\varphi^{\prime}$ is an isomorphism. We claim that there exists a homomorphism $f: F /\left[R V^{*} F\right] \rightarrow F / S_{1}$ such that the following diagrams are commutative.

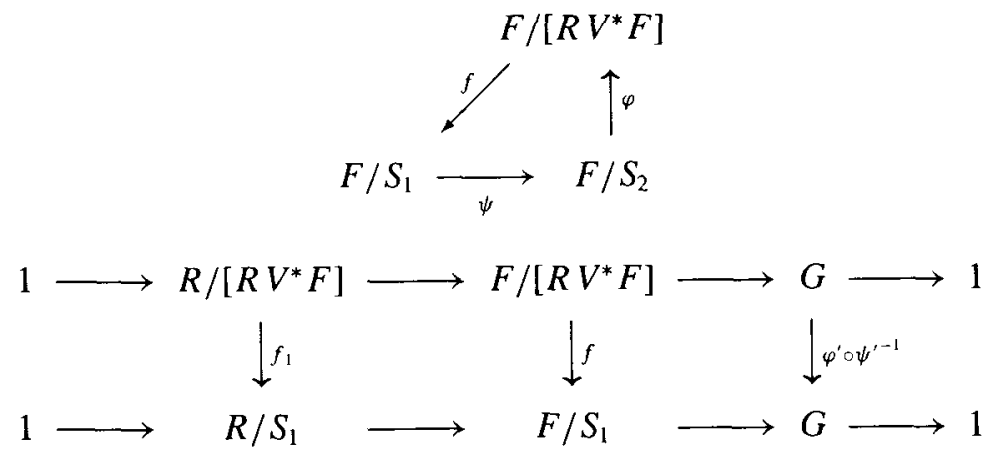

where $\psi^{\prime}: G \rightarrow G$ is induced by $\psi$, and $\varphi^{\prime} \circ \psi^{\prime-1}$ is an isomorphism. The homomorphism $f$ is obtained as follows. Since $\psi$ is surjective there is a homomorphism

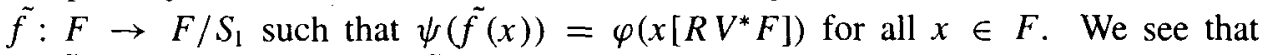
$\psi(\tilde{f}(R))=R / S_{2}$, and so $\tilde{f}(R) \subseteq \psi^{-1}\left(R / S_{2}\right)=R / S_{1}$. Since $R / S_{1} \subseteq V^{*}\left(F / S_{1}\right)$ it follows $\tilde{f}\left(\left[R V^{*} F\right]\right)$ is trivial; thus $\tilde{f}$ induces a map $f: F /\left[R V^{*} F\right] \rightarrow F / S_{1}$, as required.

Lemma 3.3 implies that $f$ is onto. Put $\operatorname{ker} f=T /\left[R V^{*} F\right]$. Then $T(R \cap V(F))=$ $R$. But $\operatorname{ker} f \subseteq \operatorname{ker} \varphi$, and hence $T \subseteq S_{2}$ and so $T=S_{2}$. Therefore $\operatorname{ker} f=\operatorname{ker} \varphi$, which implies that $\psi$ is an isomorphism.

The following corollary shows that all $\mathcal{V}$-covering groups of any group in the variety $\mathcal{V}$ are Hopfian.

COROLLARY 3.5. Let $\mathcal{V}$ be a variety of groups defined by the set of laws $V$, and $G$ be an arbitrary group of $\mathcal{V}$. Then every $\mathcal{V}$-covering group of $G$ is Hopfian.

ProOf. Let $G^{*}$ be a $\mathcal{V}$-covering group of $G$. Then there exists a normal subgroup $A$ of $G^{*}$ such that $A \subseteq V\left(G^{*}\right) \cap V^{*}\left(G^{*}\right), A \cong \mathcal{V} M(G)$, and $G^{*} / A \cong G$. Since $G$ is in the variety, it follows that $V(F) \subseteq R$, and hence $\mathcal{V} M(G)=V(F) /\left[R V^{*} F\right]$. Thus if $f: G^{*} \rightarrow G^{*}$ is an epimorphism, then $f(A)=A$; and hence by the above theorem $G^{*}$ is a Hopfian group.

\section{Acknowledgement}

The authors wish to thank the referee for his valuable suggestions, which made the paper more readable. 


\section{References}

[1] P. Hall, 'The classification of prime-power groups', J. Reine Angew. Math. 182 (1940), 130-141.

[2] N. S. Hekster, 'On the structure of $n$-isoclinism classes of groups', J. Pure Appl. Algebra 40 (1986), 63-85.

[3] — ' 'Varieties of groups and isologisms', J. Austral. Math. Soc. (Series A) 46 (1989), 22-60.

[4] M. R. R. Moghaddam, 'On the Schur-Baer property', J. Austral. Math. Soc. (Series A) 31 (1981), 343-361.

[5] M. R. R. Moghaddam and A. R. Salemkar, 'Varietal isologisms and covering groups', Arch. Math., to appear.

[6] — , 'Characterization of varietal covering and stem groups', Comm. Algebra, to appear.

[7] M. R. R. Moghaddam, A. R. Salemkar and M. M. Nasrabadi, 'Some inequalities for the Baerinvariants, and covering groups', preprint.

[8] H. Neumann, Varieties of groups (Springer, Berlin, 1967).

[9] P. M. Weichsel, 'On isoclinism', J. London Math. Soc. 38 (1963), 63-65.

Faculty of Mathematical Sciences

Ferdowsi University of Mashhad

Iran

e-mail: Moghadam@science2.um.ac.ir 